\title{
Causas y factores de riesgo asociados con la mortalidad predestete de corderos en Colima-México
}

\author{
Causes and risk factors associated with pre-weaning lamb mortality in \\ Colima, Mexico
}

\author{
Miguel García-Pérez ${ }^{1}$, Rafael Macedo-Barragán ${ }^{1,3}$, Luis García-Márquez', \\ Mauricio Valencia-Posadas ${ }^{2}$, Victalina Arredondo-Ruiz ${ }^{1}$, César García-Casillas ${ }^{1}$
}

\section{RESUMEN}

\begin{abstract}
El estudio tuvo como objetivo de estudiar las causas y factores de riesgo asociados a la mortalidad predestete en sistemas de producción de corderos bajo sistemas extensivos y semi-extensivos en un municipio de la región costera del estado de Colima, México. La mortalidad se evaluó mediante necropsia y la información sobre el número de parto y la condición corporal de la oveja antes del parto, el tamaño de la camada, el sexo, la raza y el peso del cordero se registró en el momento del parto. El análisis estadístico se hizo mediante la prueba de independencia de Chi-cuadrado y por medio de la Regresión Logística por pasos. La tasa global de mortalidad predestete fue de $27.9 \%$. El número de corderos nacidos y muertos fue de 875 y 244 , respectivamente, donde $88.1 \%$ de la mortalidad ocurrió durante las primeras 48 horas del nacimiento por el síndrome de inaniciónexposición. La mortalidad predestete se asoció significativamente $(\mathrm{p}<0.05)$ con el hato, la condición corporal de la oveja al parto y con el peso al nacimiento y la raza del cordero. Los corderos que pesaron menos de $2 \mathrm{~kg}$ al nacer $(\mathrm{OR}=8.30)$ tuvieron ocho veces más probabilidades de morir que los corderos más pesados y los corderos nacidos de ovejas con una condición corporal menor a dos al parir tuvieron más probabilidades de morir que aquellos nacidos de una oveja con mayor condición corporal $(\mathrm{OR}=3.02)$.
\end{abstract}

Palabras clave: peso al nacimiento, condición corporal, raza, sistema de producción extensivo, inanición

\author{
${ }^{1}$ Facultad de Medicina Veterinaria y Zootecnia, Universidad de Colima, Colima, México \\ ${ }^{2}$ Facultad de Medicina Veterinaria y Zootecnia, Universidad de Guanajuato, Guanajuato, México \\ ${ }^{3}$ E-mail: macedo@ucol.mx
}

Recibido: 26 de noviembre de 2020

Aceptado para publicación: 14 de mayo de 2021

Publicado: 24 de agosto de 2021

CLos autores. Este artículo es publicado por la Rev Inv Vet Perú de la Facultad de Medicina Veterinaria, Universidad Nacional Mayor de San Marcos. Este es un artículo de acceso abierto, distribuido bajo los términos de la licencia Creative Commons Atribución 4.0 Internacional (CC BY 4.0) [https:// creativecommons.org/licenses/by/4.0/deed.es] que permite el uso, distribución y reproducción en cualquier medio, siempre que la obra original sea debidamente citada de su fuente original 
The study evaluated the causes and risk factors associated with pre-weaning mortality in extensive and semi-extensive lamb production systems in a municipality in the coastal region of the state of Colima, Mexico. Mortality was assessed by necropsy and information on the number of births and the body condition of the ewe before lambing, litter size, sex, breed and weight of the lamb was recorded at the time of lambing. Statistical analysis was done using the Chi-square test of independence and by means of stepwise Logistic Regression. The overall pre-weaning mortality rate was $27.9 \%$. The number of lambs born and dead was 875 and 244 , respectively, where $88.1 \%$ of the mortality occurred during the first 48 hours of birth due to starvation-exposure syndrome. Pre-weaning mortality was significantly associated $(\mathrm{p}<0.05)$ with the flock, body condition of the ewe at parturition and with the lamb's weight at birth and breed. Lambs that weighed less than $2 \mathrm{~kg}$ at birth were eight times more likely to die than heavier lambs (OR $=8.30$ ) and lambs born to ewes with a body condition of less than two at lambing were more likely to die than those born of a sheep with higher body condition $(\mathrm{OR}=3.02)$.

Key words: birth weight, body condition, breed, extensive production system, starvation

\section{INTRODUCCIÓN}

En México, la producción de ovinos tropicales se realiza principalmente en sistemas de producción semi-extensivos y extensivos. La base de la alimentación animal en los primeros es el pastoreo de pastizales nativos y cultivados, así como con residuos de cultivos, existiendo una oferta limitada de suplementos. En los sistemas extensivos, la alimentación de los animales se basa exclusivamente en los pastizales sin recibir algún tipo de suplemento, el pastoreo se realiza sin control y no se considera la carga animal ni la disponibilidad de forraje (Vázquez-García, 2013; Muñoz-Osorio et al., 2016).

Uno de los problemas que más limita y amenaza la viabilidad económica de los sistemas de producción ovina lo constituye la mortalidad neonatal de los corderos. La tasa de mortalidad neonatal puede llegar hasta el $28 \%$ en los sistemas de producción intensivos (Mellado et al., 2016) y más del 30\% en los sistemas extensivos (Macedo et al., 2010), siendo además una situación inaceptable desde el punto de vista del bienestar animal (Dwyer, 2008).
De acuerdo con Macedo et al. (2010), la mortalidad en el estado de Colima se asocia significativamente con el sistema de producción y en donde, el síndrome de inaniciónexposición y los traumatismos constituyeron la principal causa de las pérdidas neonatales. Por otra parte, se observa una relación entre la mortalidad neonatal y otros factores predisponentes como el sexo, el peso al nacimiento y la raza del cordero, así como la condición corporal y el número de parto de la oveja, aspectos que aún no han sido ampliamente estudiados. Debido a esto, el presente estudio tuvo como objetivo estudiar los factores de riesgo asociados con la mortalidad neonatal en corderos criados en sistemas extensivos y semi-extensivos en Tecomán, Colima, México.

\section{Materiales y Métodos}

El estudio se realizó entre agosto de 2015 y julio de 2017 en el municipio de Tecomán, Colima, México, el cual cuenta con un clima cálido subhúmedo con lluvias en verano (julio-octubre) y una época seca de noviembre a junio. La precipitación y tempe- 
ratura media anual son de $750 \mathrm{~mm}$ y $26^{\circ} \mathrm{C}$, respectivamente (INEGI, 2011). Se estudiaron tres hatos seleccionados por la representatividad de los sistemas de producción ovina de la región costera del estado de Colima y por el compromiso de los productores.

- Hato A. Compuesto por 850 ovejas y 23 sementales de las razas Pelibuey, Pelifolk, Blackbelly y Dorper, criados bajo un sistema de producción extensivo. Las ovejas y sus crías pastorean de 08:00 a 18:00 h en praderas irrigadas, compuestas principalmente de pastos nativos con algunos potreros de pastos de Tanzania (Panicum maximum cv. Tanzania) y pasto Massai (Panicum maximum c.v. Massai). En las noches eran recogidas en un corral de manejo parcialmente techado. No se ofrecía complemento alimenticio y disponían de agua limpia y sal común ad libitum. Los corderos son destetados entre los 90 y 120 días de edad. Los animales mayores de un mes eran desparasitados cada cuatro meses.

- Hato B. Compuesto por 110 ovejas Dorper, Pelibuey y Katahdin, y dos sementales, uno Damara y otro Pelibuey, criados bajo un sistema de producción semi-extensivo. En época seca, las ovejas y corderos pastorean de 08:00 a 19:00 h en praderas irrigadas de pasto estrella (Cynodon plestoctachyus) y gramas nativas. En época de lluvias, el pastoreo se realiza de 10:00 a 19:00 h. El pastoreo se complementa con rastrojo de maíz y residuos de cultivos y se disponía de agua fresca, sal común y minerales ad libitum. Las ovejas con bajo peso corporal eran suplementadas con un concentrado para ganado lechero y los corderos lactantes eran alimentados con un pre-iniciador. Los corderos eran destetados entre los 90 y 120 días de edad. Los animales mayores de un mes eran vacunados contra enfermedades neumónicas y clostridiales dos veces al año y desparasitados cada cuatro meses.
- Hato C. Compuesto por 107 ovejas Blackbelly, Pelibuey, Katahdin y criollas, y dos sementales, uno Dorper y otro Pelibuey, criados bajo un sistema de producción semi-extensivo. Las ovejas y sus corderos pastoreaban de 08:00 a 15:00 y de 17:00 a 19:00 horas en praderas irrigadas de pasto estrella, pasto Bermuda (Cynodon dactylon), leucaena (Leucaena leucocephala) y gramíneas nativas. El pastoreo se complementaba con rastrojo de maíz y residuos de cultivos; y disponían de agua fresca, sal común y minerales ad libitum. Durante la estación seca se ofrecía una mezcla de sorgo molido y entero a las ovejas de bajo peso corporal y a las que amamantaban trillizos. Los corderos tuvieron acceso a un alimento pre-iniciador para lechones y fueron destetados entre los 90 y 120 días de edad. Los animales mayores de un mes fueron desparasitados y vacunados contra enfermedades neumónicas y clostridiales dos veces al año.

Al momento del parto se identificó a la oveja y a la cría (una o más), y se registró el sexo, raza y peso. Para el control de peso se utilizaron balanzas digitales de similar precisión en las tres fincas. Además, se registró el número de parto y la condición corporal de la oveja previa al parto de acuerdo con la metodología descrita por Russel et al. (1969).

Se hizo la necropsia a los corderos muertos, tanto in situ como en el Laboratorio de Patología de la Universidad de Colima. Se tomaron muestras de órganos con lesiones, se fijaron en formol amortiguado al $10 \%$ $\mathrm{y}$, posteriormente, fueron procesados siguiendo el procedimiento habitual de la técnica histológica (infiltración, inclusión y corte). Se hicieron cortes de 5 ìm que fueron teñidos con hematoxilina-eosina (Prophet et al., 1992; McGavin y Zachary, 2011). 
Cuadro 1. Causas de mortalidad predestete en corderos criados bajo manejo semi-extensivo extensivo en Colima, México

\begin{tabular}{lcc}
\hline \multirow{2}{*}{ Causa } & \multicolumn{2}{c}{ Corderos muertos } \\
\cline { 2 - 3 } & $\mathrm{n}$ & $\%$ \\
\hline Inanición - & 215 & 88.1 \\
exposición & & \\
Depredación & 10 & 4.1 \\
Sin diagnóstico & 7 & 2.9 \\
Distocia & 7 & 2.9 \\
Neumonía & 3 & 1.2 \\
Traumatismo & 2 & 0.8 \\
\hline Total & 244 & 100.0 \\
\hline
\end{tabular}

*Corderos nacidos a término, pero muertos sin síntomas o lesiones propias de inaniciónexposición, distocia o enfermedad infecciosa

Para los análisis estadísticos se empleó el software Statgraphics Centurion XVI. Se realizó una prueba de independencia de Chicuadrado para analizar la asociación entre el hato $(\mathrm{A}, \mathrm{B}, \mathrm{C})$, condición corporal $(<1,1.1$ $1.9,2-2.9, \geq 3)$ y número de parto $(1,2,3,4,5$, $>6)$ de la oveja, tamaño de camada $(1,2,3$, 4), raza (Pelibuey, Dorper, Pelifolk, Blackbelly, Katahdin, cruza), sexo (macho, hembra) y peso al nacimiento $(<1.0,1.0-1.9,2.0-2.9$, $\geq 3.0 \mathrm{~kg}$ ) del cordero con la mortalidad neonatal. Además, se realizó un análisis de Regresión Logística (Hosmer et al., 2013), incluyendo aquellos factores que resultaron asociados con la mortalidad ( $\mathrm{p}<0.05$ ). Para ello, los datos se reagruparon de la siguiente forma: hato (A, B y C), condición corporal de la oveja $(<2.0, \geq 2.0)$, peso al nacimiento del cordero $(<2.0, \geq 2.0)$, raza del cordero (pura, cruza) (Hosmer et al., 2013).
Resultados y Discusión

La tasa global de mortalidad predestete fue de $27.9 \%$. El número de corderos nacidos y muertos durante el periodo de estudio fue de 875 y 244 , respectivamente, El $88.1 \%$ murió durante las primeras 48 horas de nacidos a causa del síndrome de inanición-exposición (Cuadro 1). Los hallazgos indicativos de este síndrome fueron la falta de calostro en el abomaso, la presencia de meconio en el tracto intestinal y la ausencia de tejido adiposo pericárdico y perineal.

La mortalidad predestete se asoció significativamente con la condición corporal de la oveja al parto. La tasa de mortalidad fue mayor $(p<0.05)$ entre los corderos nacidos de una oveja con una condición corporal menor a uno, en tanto que a medida que la condición corporal aumentó la mortalidad de los corderos disminuyó. El hato tuvo un efecto significativo sobre la tasa de mortalidad, siendo mayor $(p<0.05)$ en el lote A en comparación con los lotes B y C. No se encontró asociación significativa entre la mortalidad predestete y el número de parto o tamaño de camada (Cuadro 2).

La raza y el peso al nacimiento se asociaron significativamente con la mortalidad de los corderos. Los corderos de raza pura mostraron una mayor tasa de mortalidad $(\mathrm{p}<0.05)$ que los corderos cruzados, en tanto que los corderos con un peso menor a $1 \mathrm{~kg}$ fueron más susceptibles a morir $(\mathrm{p}<0.05)$ que los corderos nacidos de mayor peso. Por otra parte, el sexo del cordero no influyó la mortalidad predestete (Cuadro 3).

De acuerdo con el análisis de regresión logística (Cuadro 4), la razón de momios para el hato de nacimiento fue de 0.18 , con un intervalo de confianza menor a 1 , por lo que los corderos nacidos en los rebaños $\mathrm{B}$ y C tuvieron un riesgo de muerte $82 \%$ menor que los nacidos en el hato A. Los corderos con un 
Cuadro 2. Asociación entre la mortalidad predestete con el hato, tamaño de camada y la condición corporal, número de parto de la oveja

\begin{tabular}{lccccc}
\hline Variable & Nivel & $\begin{array}{c}\text { Corderos } \\
\text { nacidos }\end{array}$ & $\begin{array}{c}\text { Corderos } \\
\text { muertos }\end{array}$ & $\begin{array}{c}\text { Mortalidad } \\
(\%)\end{array}$ & Valor $p$ \\
\hline Hato & $\mathrm{A}$ & 727 & 225 & 30.95 & 0.00 \\
& $\mathrm{~B}$ & 52 & 12 & 23.08 & \\
Condición & $\mathrm{C}$ & 96 & 7 & 7.29 & \\
corporal & 1 & 144 & 126 & 87.50 & 0.00 \\
& 1.1 & 464 & 89 & 19.18 & \\
& $2-1.9$ & 161 & 20 & 12.42 & \\
Número de & 2.9 & 106 & 9 & 8.49 & \\
parto & 1 & 149 & 35 & 23.48 & 0.18 \\
& 2 & 197 & 50 & 25.38 & \\
& 3 & 191 & 57 & 29.85 & \\
& 4 & 149 & 40 & 26.85 & \\
Tamaño de & 5 & 110 & 31 & 28.18 & \\
camada & 1 & 79 & 31 & 39.24 & \\
& 2 & 409 & 112 & 27.38 & 0.81 \\
& 3 & 450 & 129 & 28.67 & \\
& 4 & 12 & 2 & 16.67 & \\
\hline
\end{tabular}

peso al nacimiento menor a $2 \mathrm{~kg}$ tuvieron ocho veces más probabilidades de morir que los corderos con un peso superior a $2 \mathrm{~kg}(\mathrm{OR}=$ 8.30) en tanto que los corderos nacidos de ovejas con una condición corporal previa al parto menor de dos tuvieron tres veces más probabilidades de morir que aquellos nacidos de ovejas una condición corporal mayor (OR =3.02). Por otra parte, la raza del cordero no representó un factor de riesgo para la mortalidad predestete.

\section{Discusión}

La tasa de mortalidad predestete fue ligeramente inferior a la encontrada en un estudio previo $(31.0 \%)$ realizado en la misma región en un hato con un manejo similar a los aquí estudiados (Macedo et al., 2010).

En línea con otros estudios, el síndrome de inanición-exposición fue la principal causa de muerte de los corderos (Macedo et al., 2010; Abdelqader et al., 2017). La ocurrencia de este síndrome está estrechamente asociada con factores relacionados con el clima como cambios repentinos de temperatura al momento del parto. También se relaciona con factores propios del cordero como son el bajo peso corporal al nacimiento, mala capacidad de succión, baja ingesta de calostro y pobre instinto materno de la oveja (Nowak et al., 2000; Sawalha et al., 2007). La cría utiliza al nacer sus escasas reservas de energía para mantener el calor, y en caso de no recibir 
Cuadro 3. Asociación entre la mortalidad predestete con el tamaño de camada y el sexo, peso al nacimiento y raza del cordero

\begin{tabular}{|c|c|c|c|c|c|}
\hline Variable & Nivel & $\begin{array}{c}\text { Corderos } \\
\text { nacidos }\end{array}$ & $\begin{array}{l}\text { Corderos } \\
\text { muertos }\end{array}$ & $\begin{array}{c}\text { Mortalidad } \\
(\%)\end{array}$ & Valor $p$ \\
\hline \multirow[t]{2}{*}{ Sexo } & Macho & 471 & 138 & 29.30 & \multirow[t]{2}{*}{0.31} \\
\hline & Hembra & 404 & 106 & 26.24 & \\
\hline \multirow{4}{*}{$\begin{array}{l}\text { Peso al } \\
\text { nacimiento } \\
(\mathrm{kg})\end{array}$} & $<1.00$ & 62 & 52 & 83.87 & \multirow[t]{4}{*}{0.00} \\
\hline & $1.00-1.99$ & 509 & 165 & 32.42 & \\
\hline & $2.00-2.99$ & 189 & 15 & 7.94 & \\
\hline & $\geq 3.00$ & 115 & 12 & 10.43 & \\
\hline \multirow[t]{6}{*}{ Raza } & Pelibuey & 269 & 89 & 33.09 & \multirow[t]{6}{*}{0.00} \\
\hline & Dorper & 142 & 43 & 30.28 & \\
\hline & Pelifolk & 165 & 48 & 29.09 & \\
\hline & Blackbelly & 186 & 52 & 27.96 & \\
\hline & Katahdin & 9 & 0 & 0 & \\
\hline & Cruza & 104 & 12 & 11.54 & \\
\hline
\end{tabular}

Cuadro 4. Análisis de regresión logística de los factores de riesgo asociados con la mortalidad predestete de corderos en Colima, México

\begin{tabular}{lccccccc}
\hline \multirow{2}{*}{ Variable } & Nivel & \multicolumn{2}{c}{ Corderos muertos } & \multirow{2}{*}{$\beta$} & $P$ & $\mathrm{R} M$ & IC 95\% \\
\cline { 2 - 4 } & $\mathrm{A}$ & 225 & 30.95 & & & & \\
\hline Hato & $\mathrm{B}, \mathrm{C}$ & 19 & 12.84 & -1.71 & 0.00 & 0.18 & $0.06-0.59$ \\
& $<2.0$ & 217 & 38.00 & & & & \\
Peso al & $\geq 2.0$ & 27 & 8.88 & 2.12 & 0.00 & 8.30 & $4.54-15.19$ \\
nacimiento & & & & & & & \\
(kg) & $<2.0$ & 215 & 35.36 & & & & \\
Condición & $\geq 2$ & 29 & 10.86 & 1.11 & 0.04 & 3.02 & $1.06-8.60$ \\
corporal & Pura & 232 & 30.09 & & & & \\
Raza & Cruza & 12 & 11.54 & 0.70 & 0.18 & 2.01 & $0.73-5.57$ \\
\hline
\end{tabular}

$\beta$ : coeficiente; RM: razón de momios 
calostro dentro de las 2-3 horas posteriores al nacimiento, estas reservas se agotan y se produce hipotermia (Dwyer et al., 2016). Debido a que inmediatamente después del nacimiento, el cordero depende completamente de su madre la presencia de este síndrome está estrechamente relacionada con el manejo que se realice en la unidad de producción. Otros autores señalan como principales causas de mortalidad predestete a los cuadros de septicemia, neumonía e infecciones gastrointestinales causadas principalmente por Pasteurella sp, Salmonella sp, Escherichia coli, Toxoplasma gondii, Cryptosporidium sp y Clostridium perfringes, entre otros (Nóbrega et al., 2005; Woldemariam et al., 2014; Holmøy et al., 2017).

La asociación entre la mortalidad y el hato se explica por el manejo de la alimentación implementado en cada unidad productiva. Las ovejas del hato A fueron alimentadas exclusivamente con pasto y no recibieron ningún alimento complementario, lo que es característico de los sistemas de producción extensiva. En general, los pastos tropicales tienen un alto contenido de fibra y un bajo contenido de proteína y densidad energética, por lo que no cumplen con los requisitos nutricionales de las ovejas, especialmente durante la última etapa de la gestación y la lactancia temprana (Da Costa et al., 2014). En el caso de los rebaños B y C, los corderos recibieron un alimento pre-iniciador y las ovejas gestantes con bajo peso corporal y aquellas amamantando trillizos recibieron una suplementación y, en consecuencia, tuvieron un menor riesgo de muerte por inanición del cordero, lo cual concuerda con el estudio de Binns et al. (2002). Para el tamaño del hato, Holmøy y Waage (2015) encontraron que la tasa de mortalidad neonatal fue mayor que en los rebaños más grandes $(>250$ ovejas paridas) que los rebaños con menos de 26 ovejas; sin embargo, el mismo grupo de autores no revelaron asociación significativa entre las pérdidas de corderos y el tamaño del hato en otros estudios (Holmøy et al., 2014).
Christley et al. (2003) encontraron tasas de mortalidad en corderos más altas a medida que disminuye la condición corporal de las madres. La baja condición corporal registrada por una gran proporción de las ovejas estudiadas fue consecuencia de una mala nutrición durante las distintas etapas de la gestación. Una nutrición deficiente durante la gestación no solo afecta negativamente el peso al nacimiento del cordero y la condición corporal de la oveja al momento del parto, también deprime el desarrollo de la ubre y la producción de calostro, dando como consecuencia un aumento de la mortalidad de los corderos (Nowak, 1996). Asimismo, las ovejas con una mala condición corporal tienden a buscar alimento poco después del parto, abandonando o permaneciendo menos tiempo al cuidado del cordero (Barros de Moraes et al., 2016). Al-Sabbagh et al. (1995) no encontraron efecto negativo sobre la mortalidad de los corderos cuando la condición corporal de la oveja al parto fue entre 2.5 y 3.5. Se reconoce que corderos con bajo peso al nacer $(<2 \mathrm{~kg})$ están predispuestos a morir por inanición e hipotermia por no haber ingerido el calostro en las primeras horas de vida (Nowak y Poindron, 2006).

Estudios recientes han registrado una mayor tasa de mortalidad en corderos de raza pura en comparación con corderos cruzados (Mustafa et al., 2014; McHugh et al., 2016), posiblemente debido el efecto de la heterosis (Gama et al., 1991). Por otra parte, y coincidiendo con los hallazgos de Turkson y Sualisu (2005) y Mandal et al. (2007), el sexo de los corderos no tuvo relación significativa con la tasa de mortalidad neonatal; no obstante otros investigadores señalan una mayor mortalidad en corderos machos (Sawalha et al., 2007; Abdelqader et al., 2017). A pesar de que el efecto de los factores ligados al sexo sobre la mortalidad de los corderos no es claro, Warren y Mysterud (2005) observaron que el comportamiento del cordero macho puede hacerlo más susceptibles a la depredación ya que se alejan más de la madre y del rebaño, 
en tanto que Dwyer (2003) señala que los corderos machos Suffolk eran más lentos para amantarse que las hembras.

Si bien el número de parto de la oveja no influyó sobre la mortalidad del cordero, tal y como se reporta en otros estudios (Warren y Mysterud, 1995; Mandal et al., 2007), McHugh et al. (2016) encontraron que los corderos de ovejas primíparas tenían un mayor riesgo de morir en comparación con los corderos de ovejas multíparas, debido principalmente por una mayor dificultad para parir.

En el presente estudio no se encontró un efecto del tamaño de camada sobre la mortalidad de los corderos; sin embargo, los mellizos a menudo se encuentran en desventaja por un menor peso al nacimiento, menores reservas de energía y menor consumo de calostro que los corderos únicos (Nowak y Poindron, 2006; Abdelqader et al., 2017).

\section{Conclusiones}

- La mortalidad neonatal fue mayor entre los corderos criados bajo un sistema de producción extensivo, siendo el síndrome de inanición-exposición la principal causa de muerte.

- La mortalidad estuvo fuertemente asociada con el peso del cordero al nacimiento y a la condición corporal de la oveja al momento del parto

\section{Literatura Citada}

1. Abdelqader A, Irshaid R, Tabbaa MJ, Abuajamieh M, Titi H, Al-Fataftah R. 2017. Factors influencing Awassi lambs survivorship under fields conditions. Livest Sci 199: 1-6. doi: 10.1016/ j.livsci.2017.03.007

2. Al-Sabbagh TA, Swanson LV, Thompson JM. 1995. The effect of ewe body condition at lambing on colostral immunoglobulin $\mathrm{G}$ concentration and lamb performance. J Anim Sci 73: 28602864. doi: $10.2527 / 1995.73102860 \mathrm{x}$

3. Barros de Moraes A, Espírito CPCH, Fischer V, Fajardo N, Farias AM, Caillava da Porciuncula, G. 2016. Ewe maternal behavior score to estimate lamb survival and performance during lactation. Acta Sci 38: 327-332. doi: 10.4025/actascianimsci.v38i3.29923

4. Binns SH, Cox IJ, Rizvi S, Green LE. 2002. Risk factors for lamb mortality on UK sheep farms. Prev Vet Med 52: 87303. doi: 10.1016/s0167-5877(01)00255-0

5. Christley RM, Morgan KL, Parkin TDH, French NP. 2003. Factors related to the risk of neonatal mortality, birth-weight and serum immunoglobulin concentration in lambs in the UK. Prev Vet Med 57: 209-226. doi: 10.1016/ s0167-5877(02)00235-0

6. Da Costa TJN, Marques RA, Torreão MCA, Rocha B.L, Gottardi FP, Jácome AM, de Souza Júnior EL, Lopes OR. 2014. Concentrate supplementation during pregnancy and lactation of ewes affects the growth rate of lambs from a variety of crosses. Rev Bras Zootecn 43: 544-550. doi: 10.1590/ S1516-35982014001000006

7. Dwyer CM. 2003. Behavioural development in the neonatal lambs: effect of maternal and birth-related factors. Theriogenology 59: 1027-1059. doi: 10.1016/s0093-691x(02)01137-8

8. Dwyer CM. 2008. The welfare of the neonatal lamb. Small Ruminant Res 76: 31-41. doi: 10.1016/j.smallrumres.2007.12.011

9. Dwyer CM, Conington J, Corbiere F, Holmoy IH, Muri K, Nowak R, Rooke J, Vipond J, Gautier, JM. 2016. Invited review: improving neonatal survival in small ruminants: science into practice. Animal 10: 449-459. doi: 10.1017/S1751731115001974

10. Gama LT, Dickerson GE, Young LD, Leymaster, K.A. 1991. Effects of breed, heterosis, age of dam, litter size and birth weight on lamb mortality. J 
Anim Sci 69: 2727-2743. doi: 10.2527/ $1991.6972727 \mathrm{x}$

11. Holmoy IH, Waage S. 2015. Time trends and epidemiological patterns of perinatal lamb mortality in Norway. Acta Vet Scand 57:2-11. doi: 10.1186/s13028015-0155-6

12. Holmøy IH, Waage S, Granquis, EG, L'Abée-Lund TM, Ersdal C, Hektoen L, Sorby R. 2017. Early neonatal lamb mortality: postmortem findings. Animal 11: 295-305. doi: 10.1017/S175173111$600152 \mathrm{X}$

13. Holmøy IH, Waage S, Gröhn YT. 2014. Ewe characteristics associated with neonatal loss in Norwegian sheep. Prev Vet Med 114: 267-275. doi: 10.1016/ j.prevetmed.2014.02.007

14. Hosmer Jr DW, Lemeshow S, Sturdivant RX. 2013. Applied logistic regression. New Jersey, USA: John Wiley. $375 \mathrm{p}$.

15. [INEGI] Instituto Nacional de Estadística y Geografía. 2011. Mapas. Colima. [Internet]. Disponible en: http:// internet.contenidos.inegi.org.mx/contenidos/Productos/prod_serv/contenidos/ espanol/bvinegi/productos/geografia/ conden_estatles/col/702825003569.pdf

16. Macedo R, Arredondo V, Rodríguez J, Ramírez J, López B. 2010. Efecto del sistema de producción, de la época de nacimiento y del sexo sobre la mortalidad neonatal de corderos Pelibuey. Trop Subtrop Agroecosyt 12: 77-84.

17. Mandal A, Prasad H, Kumar A, Roy R, Sharma N. 2007. Factors associated with lamb mortalities in Muzaffarnagari sheep. Small Ruminant Res 71:273-279. doi: 10.1016/J.SMALLRUMRES.2006.07.010

18. McGavin MD, Zachary JF. 2011. Pathologic basis of veterinary disease. $5^{\text {th }}$ ed. London, UK: Elsevier. $1394 \mathrm{p}$.

19. McHugh N, Berry DP, Pabiou T. 2016. Risk factors associated with lambing traits. Animal 10: 89-95. doi: $10.1017 / \mathrm{S} 1751731115001664$
20. Mellado M, Macías U, Avendaño L, Mellado J, García, J.E. 2016. Growth and pre-weaning mortality of Katahdin lamb crosses. Rev Colomb Cienc Pec 29: 288-295. doi: 10.17533/udea.rccp.$\mathrm{v} 29 \mathrm{n} 4 \mathrm{a} 06$

21. Muñoz-Osorio GA, Aguilar-Caballero AJ, Sarmiento-Franco LA, Wurzinger M, Cámara-Sarmiento R. 2016. Technologies and strategies for improving hair lamb fattening systems in tropical regions: A review. Ecosist Recur Agropec 3: 267-277.

22. Mustafa MI, Mehmood MM, Lateef M, Bashir MK, Khalid AR. 2014. Factors influencing lamb mortality from birth to weaning in Pakistan. Pak J Life Soc Sci 12: 139-143.

23. Nóbrega Jr JE, Riet-Correa F, Nóbrega RS, Medeiros JM, Vasconcelos JS, Vilar DSS, Tabosa IM. 2005. Mortalidade perinatal de cordeiros no semi-árido da Paraíba. Pesqui Vet Bras 25: 171-178. doi: 10.1590/S0100736X2005000300008

24. Nowak R. 1996. Neonatal survival contribution from behavioral studies in sheep. Appl Anim Behav Sci 49: 61-72. doi: 10.1016/0168-1591(95)00668-0

25. Nowak R, Poindron P. 2006. From birth to colostrum: early steps leading to lamb survival. Reprod Nutr Dev 46: 431-446. doi: 10.1051/rnd:2006023

26. Nowak R, Porter RH, Lévy F, Orgeur $P$, Schaal, B. 2000. Role of mother-young interactions in the survival of offspring in domestic mammals. Rev Reprod 5: 153163. doi: 10.1530/ror.0.-0050153

27. Prophet E, Mills B, Arrington J, Sobin, L. 1992. Laboratory methods in histotechnology. Washington: Armed Forces, Institute of Pathology. $279 \mathrm{p}$.

28. Russel AJF, Doney JM, Gunn RG. 1969. Subjective assessment of body fat in live sheep. J Agric Sci 72: 451-454.

29. Sawalha RM, Conington J, Brotherstone S, Villanueva V. 2007. Analyses of lamb survival of Scottish Blackface sheep. Animal 1: 151-157. doi: 10.1017/ S1751731107340056 
30. Turkson PK, Sualisu M. 2005. Risk factors for lamb mortality in Sahelian sheep on a breeding station in Ghana. Trop Anim Health Pro 37: 49-64. doi: 10.1023/b:trop.0000047935.78168.46.

31. Vázquez-García V. 2013. Sheep Production in the mixed-farming systems of Mexico: where are the women? Rangelands 35: 41-46. doi: 10.2111/ RANGELANDS-D-13-00029.1
32. Warren JT, Mysterud, I. 1995. Mortality of domestic sheep in freeranging flocks in southeastern Norway. JAnim Sci 73: 1012-1018. doi: 10.2527/ 1995.7341012x

33. Woldemariam S, Zewde S, Hameto D, Habtamu, A. 2014. Major causes of lamb mortality at Ebinat Woreda, Amhara National Regional State, north-western Ethiopia. Ethiop Vet J 18: 57-71. 